\title{
Correction to: Porous yttria-stabilized zirconia ceramics with low thermal conductivity via a novel foam-gelcasting method
}

Xuanyu Meng ${ }^{1,2}$, Jie $\mathrm{Xu}^{1,2, *}$ (D), Jiatong Zhu ${ }^{1,2}$, Jia Zhao ${ }^{1}$, Zhuolun $\mathrm{Li}^{1}$, Emilia Pawlikowska ${ }^{4}$, Mikołaj Szafran ${ }^{3}$, and Feng Gao ${ }^{1}$

${ }^{1}$ State Key Laboratory of Solidification Processing, MIIT Key Laboratory of Radiation Detective Material and Device, NPU-QMUL Joint Research Institute of Advanced Materials and Structures (JRI-AMAS), School of Material Science and Engineering, Northwestern Polytechnical University, Xi'an 710072, China

${ }^{2}$ Research and Development Institute of Northwestern, Polytechnical University in Shenzhen, Shenzhen 518057, China

${ }^{3}$ Faculty of Chemistry, Warsaw University of Technology, Noakowskiego 3, 00-664 Warsaw, Poland

${ }^{4}$ Institute of High Pressure Physics of the Polish Academy of Sciences, ul. Sokolowska 29/37, 01-142 Warsaw, Poland

Published online:

3 January 2022

(C) Springer Science+Business Media, LLC, part of Springer

Nature 2021

J Mater Sci (2020) 55:15106-15116 https://doi.org/10.1007/s10853-020-04900-3

In the original publication the author affiliation for Emilia Pawlikowska was incorrect. It is correct here.
Publisher's Note Springer Nature remains neutral with regard to jurisdictional claims in published maps and institutional affiliations.

The original article can be found online at https:/ / doi.org/10.1007/s10853-020-04900-3.

Address correspondence to E-mail: xujie@nwpu.edu.cn 